\title{
The Blazar Spectral Sequence and GLAST
}

\author{
L. Maraschi, G. Ghisellini and F. Tavecchio \\ INAF-Osservatorio Astronomico di Brera, Milano, Italy
}

\begin{abstract}
The present status and understanding of the "spectral sequence" of blazars is discussed in the perspective of the upcoming GLAST launch. The vast improvement in sensitivity will allow to i) determine more objectively the "average" gamma-ray properties of classes objects ii) probe more deeply the ratio between accretion power and jet power in different systems.
\end{abstract}

Keywords: Gamma-rays - Relativistic jets - Galaxies: active

PACS: $95.85 . \mathrm{Pw}$; $98.54 . \mathrm{Cm}$

\section{INTRODUCTION}

The spectral sequence of blazars (Fossati et al.,1998 al.,1998) was constructed merging three complete blazar samples (two radio selected, one X-ray selected: 2 Jy FSRQ, Wall \& Peacock 1985, 1Jy BL Lac, Kuhr et al. 1981, and Slew Survey BL Lac, Elvis et al. 1992), grouping all the objects in radio luminosity bins and averaging monochromatic luminosities of objects within each radio-luminosity bin. The procedure is thus prone to various biases (Maraschi \& Tavecchio 2001), in particular the gamma-ray data were largely incomplete.

The resulting "sequence" shows that the blazar SEDs are double humped and that the two peaks shift to higher energies with decreasing luminosity. Systematic modelling of the SEDs of individual objects (Ghisellini et al. 1998) yields basically uniform beaming factors and jet parameters varying along the sequence in the sense of an increasing energy density and decreasing electron critical energy at higher luminosities. Thus the "sequence" offers a suggestive indication that the basic spectral properties of blazar jets could be related to the different powers involved and possibly represent an evolutionary sequence in cosmic history (Boettcher and Dermer 2002; Cavaliere and D'Elia 2002).

The validity of the sequence concept has been questioned on the basis of deeper and larger blazar surveys (e.g. Giommi et al. 2005, Padovani 2007) which however lack until now the very important gamma-ray data.

Here we wish to address two points. The first concerns the validity of the original claim within the presently known bright blazar SEDs, the second concerns an anticipation of the types of blazars that may be detected by GLAST.

\section{NEW DATA / NEW SOURCES}

Given the limited space we will illustrate our points schematically, commenting few representative figures. All the figures will have in the background the double humped lines interpolating the blazar spectral sequence. The latter are just polinomial expressions connecting the average monochromatic luminosities obtained as described above.

The SED of a new high redshift FSRQ serendipitously discovered by SWIFT (BAT) J0746+2548 ( $\mathrm{z}=2.979$ ) (Sambruna et al. 2006) is shown in Fig. 1a. Clearly J0746 is extremely luminous and conforms well to the sequence, possibly suggesting a gamma-ray peak at Mev energies. The spectral shape in the gamma-ray band that will be measured by GLAST for a large number of blazars will provide an essential information to constrain the position of the high energy peak of blazar SEDs thus probing the sequence concept.

3C 454.3 is a highly variable FSRQ ( $\mathrm{z}=0.859)$ already detected in gamma-rays by EGRET. The data for a "normal" state (Tavecchio et al. 2007) are shown in Fig. 1b. This source could be detected with GLAST at $1 \%$ the intensity level shown in the figure which is the average of EGRET measurements. The source underwent a strong outburst recently and was observed by SWIFT (BAT) and INTEGRAL up to more than $100 \mathrm{keV}$ (Pian et al. 2006, Giommi et al. 2006). In the latter state the expected gamma-ray flux could have been an order of magnitude brighter than detected by EGRET. A source with an intrinsically similar jet could then be detected in gamma-rays even if the jet was at a larger angle to the line of sight. The thick lines in Fig. 2 represent the model used to describe the "normal" state of 3C 454.3, computed for different viewing angles. The gamma-ray emission could be detected by GLAST up to an angle 

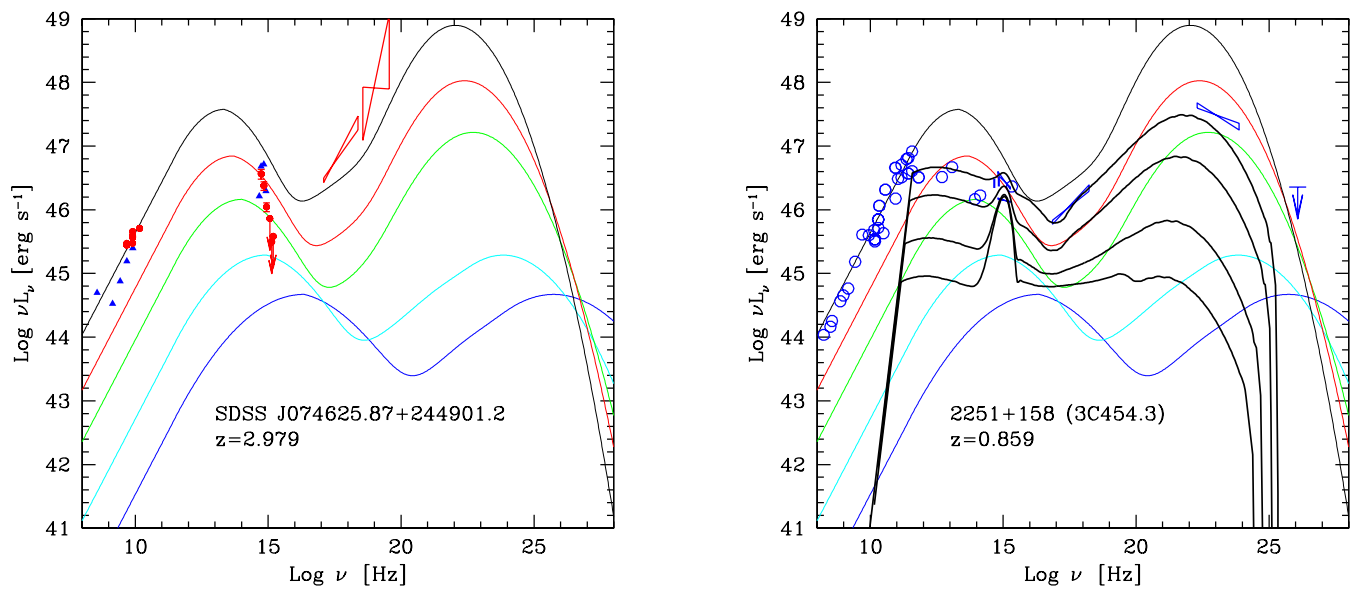

FIGURE 1. Spectral Energy Distribution of the blazars J0746+2548 (left, from Sambruna et al. 2006) and 2251+158 (right, from Tavecchio et al. 2007) overimposed on the curves interpolating the blazar sequence. For $2251+158$ we also report the model used to reproduce the data (upper black curve) and the emission expected for a misaligned jet with angles of respectively 6,8 and 10 degrees (from top to bottom).

of 10 degrees to the jet axis. In this case the SED would be significantly different than expected from the sequence, simply because the jet emission is less beamed and less prominent with respect the SED of the accretion disk, included here as a blackbody component plus a Seyfert like X-ray component. The sequence is not expected to extend to objects with jets seen at intermediate angles. The different Doppler factor causes only a linear shift of the peak position but a dramatic change in luminosiy.

Fig. 2 is devoted to blazars with lower luminosities. This part of the sequence is populated exclusively by BL Lac objects defined as HBLs due to their SEDs peaking at high energies, in the X-ray and TeV bands. In Fig. 2a the data for the "normal" state of PKS 2155-304 are plotted in green. They are well consistent with the sequence. The multifrequency data obtained during the exceptional TeV flare observed from this source in July August 2006 are also shown (see Foschini et al. 2007). During the outburst the two emission peaks do not appear to shift much in frequency but the luminosities increase by a large factor (for a short time) especially in the TeV band. Thus the high state SED deviates remarkably from the sequence expectations. For these objects, though relatively weak at $\mathrm{GeV}$ energies, GLAST observations will be important to define the shape of the high energy peak and its possible evolution during outbursts.

Finally, in Fig. 2b we show the data for $1629+4008$ ( $\mathrm{z}=0.272$ ), a blazar with an emission peak between the UV and the X-ray band discovered within a survey aimed at finding objects with anomalous properties (Padovani et al. 2002). The SED of this source complies reasonably well with the sequence expectation for an HBL, however this object shows emission lines which is not the case for HBLS. In fact the sequence included only X-ray selected BL Lacs, but no X-ray selected radio-loud objects with emission lines, as no such complete sample was available at the time (see Wolter \& Celotti 2001).

This source indicates that jets with SEDs peaking at high energies can occur in emission line AGNs. This is a new result, which however does not break the correlations inferred from the sequence, as it occurs in the low luminosity range. The question then is: what distinguishes HBLs from objects like 1629? Why emission lines are completely absent in HBLs but present in 1629 whose jet is of comparable luminosity? According to our ideas (Maraschi 2001, Maraschi \& Tavecchio 2003) HBL should accrete at highly subEddington rates, therefore in the radiatively inefficient accretion (RIAF) regime, while 1629, which shows emission lines, should be in the "standard" accretion disk regime, therefore near to its Eddington limit. This in turn implies that this source contains a central black hole of relatively modest mass. From the accretion luminosity, assuming that it corresponds to 0.1 the Eddington luminosity we can infer a mass of $6 \times 10^{7}$ solar masses. More direct estimates of the black hole mass are needed to confirm this prediction. 

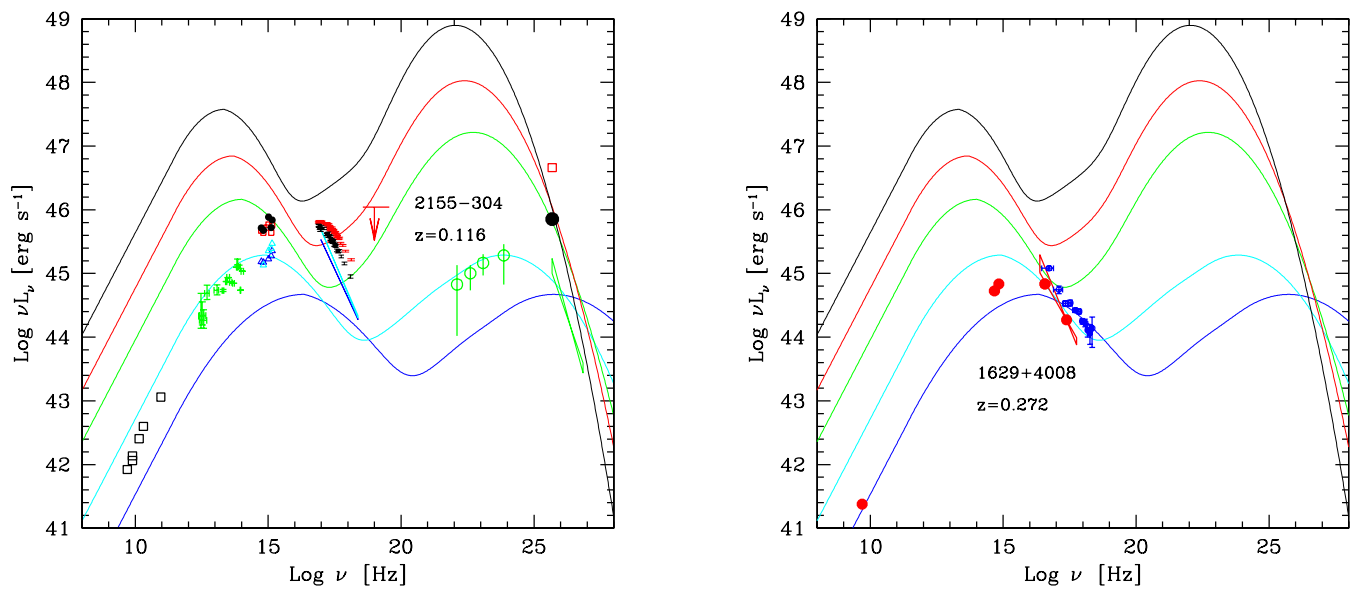

FIGURE 2. SEDs of the blazars 2251-304 (left, Foschini et al. 2007) and 1629+4008 (Padovani et al. 2002) overimposed on the blazar sequence interpolations. For PKS 2155-304 a normal state is shown together with optical/X-ray and TeV data during the exceptional outburst of July-August 2006

\section{CONCLUSIONS}

The few examples discussed above are meant to indicate how the concept of a spectral sequence for blazars, based on averages over limited samples involving only the brightest objects of each class, may be probed by GLAST. In particular, strong emphasis has been put in the past on BL Lac objects, neglecting the X-ray selected counterparts of FSRQ which may also be gamma-ray emitters. GLAST is expected to produce extraordinary advances in this field. It will increase by orders of magnitude the number of objects with measured gamma-ray flux (see Dermer these proceedings) thus allowing to study deeper and differently selected samples. These will certainly contain "mixed" objects in which the jet emission is less prominent in comparison to other AGN properties. The new gamma-ray populations should carry great potential for understanding the link between accretion power and the production of jets in extragalactic objects.

\section{REFERENCES}

1. Böttcher, M., \& Dermer, C. D. 2002, ApJ, 564, 86

2. Cavaliere, A., \& D'Elia, V. 2002, ApJ, 571, 226

3. Elvis, M., Plummer, D., Schachter, J., \& Fabbiano, G. 1992, ApJS, 80, 257

4. Foschini, L., et al. 2007, ApJ, 657, L81

5. Fossati, G., Maraschi, L., Celotti, A., Comastri, A., \& Ghisellini, G. 1998, MNRAS, 299, 433

6. Ghisellini, G., Celotti, A., Fossati, G., Maraschi, L., \& Comastri, A. 1998, MNRAS, 301, 451

7. Giommi, P., et al. 2006, A\&A, 456, 911

8. Giommi, P., Piranomonte, S., Perri, M., \& Padovani, P. 2005, A\&A, 434, 385

9. Kuehr, H., Witzel, A., Pauliny-Toth, I. I. K., \& Nauber, U. 1981, A\&AS, 45, 367

10. Maraschi, L., \& Tavecchio, F. 2003, ApJ, 593, 667

11. Maraschi, L. 2001, AIP Conf. Proc. 586: 20th Texas Symposium on relativistic astrophysics, 586, 409

12. Maraschi, L., \& Tavecchio, F. 2001, ASP Conf. Ser. 234: X-ray Astronomy 2000, 234, 437

13. Padovani, P., in "The Multi-messenger approach to high energy gamma-ray sources", 2007, in press (astro-ph/0610545)

14. Padovani, P., Costamante, L., Ghisellini, G., Giommi, P., \& Perlman, E. 2002, ApJ, 581, 895

15. Pian, E., et al. 2006, A\&A, 449, L21

16. Sambruna, R. M., et al. 2006, ApJ, 646, 23

17. Tavecchio, F., et al. 2007, ApJ, in press (astro-ph/0703359)

18. Wall, J. V., \& Peacock, J. A. 1985, MNRAS, 216, 173

19. Wolter, A., \& Celotti, A. 2001, A\&A, 371, 527 